\title{
VẬN DỤNG KỸ THUẠTT DẠY - HỌC KWLH NHÀM PHÁT HUY TÍNH CHỦ ĐộNG, SÁNG TẠO CỦA SINH VIẾN TRONG QUÁ TRİNH HỌC TẬP THEO TIÊU CHUẨN 8 CỦA CDIO Đinh Thị Yến ${ }^{(*)}$
}

(*) Thạc sĩ. Truòng Đại học Thủ Dầu Một. Email: yendt@tdmu.edu.vn

DOI: $10.37550 /$ tdmu.CFR/2021.01.122

\section{Tóm tắt}

Kỹ thuật dạy - học KWLH là tên được viết tắt các tì̀ tiếng Anh: Know, Want to Know, Learned và How to learn more. Kỹ thuật này được đề xuất bởi Donna Ogle vào năm 1986, nhằm cải thiện quá trình dạy - học, đặc biệt là việc huóng dẫn sinh viên học tập chu động, tụ nghiên cứu tài liệu. Dựa trên trải nghiệm kỹ thuật này trong giảng dạy các học phần, bài viết này chúng tôi phân tích các bước thục hiện kỹ thuật KWLH trong quá trình dạy - học trên lớp cũng nhu huớng dẫn sinh viên tụ họ nhằm giúp sinh viên tăng cuờng tính chủ động, tích cực trong việc học và đạt được chuẩn đầu ra theo Tiêu chuẩn 8 - Hoc tập chủ động của đề xướng CDIO.

Từ khóa: Học tập chủ động, kỹ thuật dạy - học KWLH, Vận dụng, tiêu chuẩn 8, CDIO

\section{1. Đặt vấn đề}

Trong những năm vừa qua, trường Đại học Thủ Dầu Một đã rất tích cực đổi mới phương pháp dạy học cho giảng viên. Từ cuối năm 2014 đến năm 2016, các lớp tập huấn về xây dựng chương trình đào tạo theo CDIO (Conceive - Design - Implement - Operate) đã liên tục được tổ chức. Trong 12 tiêu chuẩn của có 4 tiêu chuẩn bàn về vấn đề dạy và học nhằm đáp ứng chẩn đầu ra là $7,8,9,10$. Các tiêu chuẩn này tập trung về phương pháp dạy - học tập trải nghiệm, chủ động, tích cực: Tiêu chuẩn 7 - Trải nghiệm học tích hợp (Integrated Learning Experiences); Tiêu chuẩn 8 - Học chủ động (Active Learning); Tiêu chuẩn 9 - Phát triển giảng viên về kỹ năng CDIO (Enhancement of Faculty Competence); Tiêu chuẩn 10 - Nâng cao kỹ năng sư phạm cho giảng viên (Enhancement of Faculty Teaching Competence) [1]. Để sinh viên được trải nghiệm học tích hợp và nâng cao khả năng học tập chủ động ở tiêu chuẩn 7 và 8 , đòi hỏi giảng viên phải được đào tạo để phát triển năng lực, nâng cao kỹ năng ở tiêu chuẩn 9 và 10 . Để đạt được điều này, từ năm 2016 đến nay, Nhà trường hợp tác với trường đại học Victoria, Canada tập huấn nhằm nâng cao kỹ năng giảng dạy cho giảng viên theo chương trình ISW (Instruction Skills Workshop). Và hiện nay, các lớp tập huấn về nâng cao phương pháp giảng dạy vẫn được duy trì và chú trọng đến phương pháp giảng dạy hòa hợp tích cực để giúp sinh viên đạt được chuẩn đầu ra. 
Những khóa tập huấn nâng cao năng lực giảng viên nhằm thay đổi phương pháp dạy học chủ động, tích cực, thu hút sự tham gia của người học, luôn đề cao vị trí trung tâm của họ. Sinh viên/ người học được khuyến khích chủ động nghiên cứu, tìm hiểu, học, thực hành, hỏi và tự đánh giá, tự điều chỉnh. Giảng viên chỉ là người định hướng, tổ chức, hỗ trợ, cố vấn về nội dung, tài liệu, cách học và đánh giá. Khi lấy người học làm trung tâm, giảng viên cần xác định thế nào là quá trình học tập hiệu quả nhất. Trên cơ sở đó, giảng viên điều chỉnh các hoạt động dạy học sao cho phù hợp với năng lực, sở thích và nhu cầu của người học, giúp người học đạt được các chuẩn đầu ra theo tinh thần của Tiêu chuẩn 8 - Học tập chủ động của CDIO.

Hiện nay, có rất nhiều kỹ thuật dạy - học chủ động, tích cực gồm tổ chức hoạt động nhóm (kỹ thuật động não, thảo luận viết, tia chớp, sơ đồ tư duy, mảnh ghép, XYZ, bể cả, ổ bi, think - pair - share...), tổ chức hoạt động cá nhân (kỹ thuật $5 \mathrm{~W} 1 \mathrm{H}, \mathrm{ABC}, 3$ lần 3, 3-2-1 và KWL/KWLH...). Thiết nghĩ, nếu áp dụng một cách linh hoạt các kỹ thuật học tập này vào trong quá trình giảng dạy sẽ phát huy được tính tích cực, chủ động, sáng tạo trong quá trình học tập của sinh viên, giúp sinh viên đạt được kết qảu học tập cao nhất. Trong số các kỹ thuật dạy học tích cực, chúng tôi chọn kỹ thuật KWLH để nghiên cứu. Bản thân tác giả đặt giả thuyết rằng, kỹ thuật KWLH là một chiến lược dạy học giúp người học hoạt động hiệu quả cao, tăng tính chủ động, sáng tạo của họ. KWLH được thiết kế để người dạy hướng dẫn người học tự giác lĩnh hội tri thức qua việc giảng dạy, chia sẻ của giảng viên cũng như đọc hiểu các nguồn tài liệu và trở thành vị trí trung tâm trong quá trình dạy học. Kỹ thuật này cũng cho phép giảng viên thực hiện các bước như: đánh giá trước kiến thức nền tảng và sở thích của sinh viên; triển khai; đánh giá sau và định hướng tự nghiên cứu, tự học cho sinh viên.

\section{Tiêu chuẩn 8: học tập chủ động (active learning)}

CDIO là chữ viết tắt của các từ Hình thành ý tưởng (Conceive) - Thiết kế (Design) Triển khai (Implement) và Vận hành (Operate). Đây là phương pháp cải cách giáo dục nhằm nâng cao chất lượng đào tạo, đáp ứng yêu cầu xã hội trên cơ sở xác định chuẩn đầu ra. CDIO gồm 12 tiêu chuẩn, trong đó tiêu chuẩn 7,8 liên quan đến học tập chủ động, trải nghiệm, tiêu chuẩn 9,10 nâng cao phương pháp giảng dạy. Tất cả những hoạt động dạy - học, đánh giá đều tập trung vào chuẩn đầu ra của sinh viên [1]. Vì vậy, theo nguyên lý thiết kế giảng dạy và đánh giá đáp ứng chuẩn đầu ra, giảng viên sẽ phải nghiên cứu và triển khai các phương pháp học tập để sẽ giúp sinh viên đạt kết quả học tập cao nhất sau mỗi khóa học.

Tiêu chuẩn 8 là một trong những bước để đạt được nguyên lý theo Hình 1. Tiêu chuẩn này được mô tả rằng các phương pháp học tập chủ động sẽ thu hút sự tham gia của sinh viên một cách trực tiếp vào các hoạt động tư duy và giải quyết vấn đề. Giảng viên đặt các vấn đề, tổ chức các hoạt động nhằm thu hút sinh viên tự giác tham gia vào khám phá, ứng dụng, phân tích, và đánh giá các ý tưởng. Học tập chủ động trong các môn học dựa trên bài giảng có thể bao gồm các phương pháp như những cuộc thảo luận với bạn học hay trong nhóm nhỏ, làm demo, tranh luận, các câu hỏi về khái niệm, hay các đề án thiết kế - triển khai, thực hành nghề nghiệp, nghiên cứu tình huống (case studies), các phản hồi của sinh viên về nội dung họ đang học. Như vậy, sinh viên không chỉ học được nhiều hơn, mà họ còn tự nhận ra được họ học gì 
và học như thế nào. Quá trình siêu nhận thức này giúp làm tăng động lực của sinh viên để đạt được các chuẩn đầu ra của chương trình và hình thành thói quen học tập suốt đời. Với các phương pháp học tập chủ động, các giảng viên có thể giúp sinh viên tạo dựng mối liên hệ giữa các khái niệm chính yếu và tạo điều kiện thuận lợi áp dụng kiến thức này vào trong các hoàn cảnh mới [1]. Để đạt được tiêu chuẩn này, có rất nhiều phương pháp để triển khai và KWLH là một sự lựa chọn hiệu quả để sinh viên chủ động tham gia một cách tích cực bài học trên lớp cũng như tự giác tự học, tự nghiên cứu ở nhà.

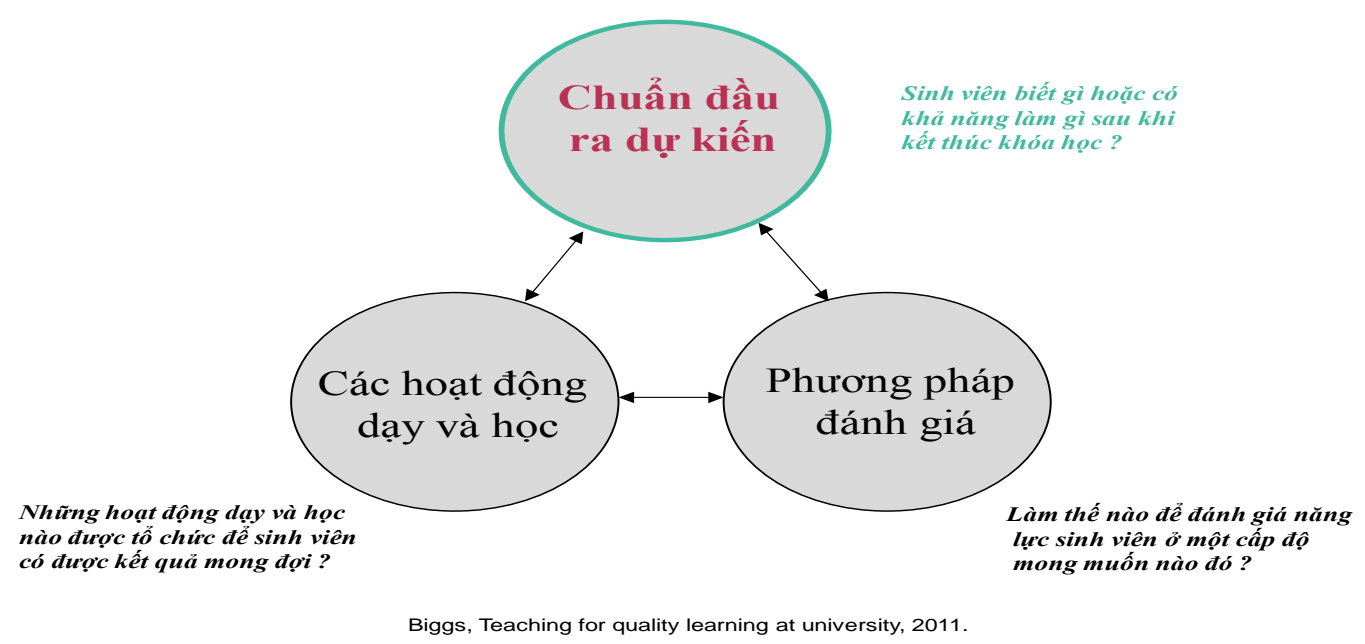

Hình 1. Nguyên lý thiết kế giảng dạy và đánh giá đáp ứng chuẩn đầu ra (Constructive Alignment)

\section{Tổng quan về kỹ thuật dạy học $\mathrm{KWL-KWLH}$}

KWL là một kỹ thuật dạy học được giới thiệu bởi Donna Ogle vào năm 1986, đây là chương trình hướng dẫn phát triển việc đọc văn bản, học chủ động bằng cách kích hoạt kiến thức nền tảng của người học. KWL gồm $\mathrm{K}$ (What $\mathrm{I}$ Know) - Những điều đã biết; $\mathrm{W}$ (What $\mathrm{I}$ Want to know) - Những điều muốn biết; $\mathrm{L}$ (What I Learned) - Những điều đã học được. Như vậy, kỹ thuật này cung cấp một cấu trúc để nhớ lại những gì người học đã biết về một chủ đề, lưu ý những gì họ muốn biết và liệt kê chính thức những gì đã học và chưa được học. Thứ tự được thực hiện gồm: Đầu tiên, người học động não tất cả những gì họ biết về chủ đề này. Thông tin liên quan được ghi lại trong cột $\mathrm{K}$ của sơ đồ KWL (Bảng 1); Thú hai, người học tạo ra một danh sách các câu hỏi về những gì họ muốn biết về chủ đề này. Những câu hỏi này được liệt kê trong cột $\mathrm{W}$; Thú $b a$, trong hoặc sau khi đọc, người học trả lời những câu hỏi họ đã nêu trong cột $\mathrm{W}$. Những gì họ đã học được sẽ ghi lại trong cột L [2].

Năm 1987, Carr \& Ogle đã sửa đổi chiến lược thành sơ đồ KWL-Plus, viết tắt của Know (Những điều đã biết), Want (Những điều muốn biết) và Learn plus Mapping and Summarizing (Những điều học được, lập bản đồ và tóm tắt). Như vậy, các nhà nghiên cứu đã bổ sung phần lập bản đồ thể hiện sự tương quan về nội dung và tóm tắt tài liệu vào chiến 
lược KWL truyền thống. Theo nhà nghiên cứu Ogle, KWL giúp người học đọc được tốt hơn các tài liệu và tương tác nhiều hơn trong quá trình dạy và học [3]. Sau khi thực hiện một số hoạt động trong các cột của KWL-Plus, người học được khuyến khích sử dụng nó như một chiến lược học tập độc lập để kích hoạt kiến thức trước đây của họ, khuyến khích họ tìm hiểu và xác định những gì họ học được. Điều này giúp họ đặt ra mục đích rõ ràng để đọc và ghi lại những gì họ đã học. Khi lập bản đồ, người học sử dụng sơ đồ tư duy - mind map và tiêu đề được đặt ở trung tâm của bản đồ, các nội dung chính trong tiêu đề là nhánh chính - nhánh cấp 1 , sau đó thêm các nhánh phụ - nhánh cấp 2 , cấp 3 với các nội dung, khái niệm để giải thích. Khi tóm tắt, người học đánh số các khái niệm trên bản đồ và viết lại thành một bản tóm tắt. Bản tóm tắt trở nên hữu ích giúp người học có thể sử dụng để tự đánh giá bản thân và người dạy có thể đánh giá lại quá trình giảng dạy.

Đến năm 1992, Ogle tiếp tục phát triển chiến lược KWL của mình, bà kết hợp KWL với các câu hỏi $5 \mathrm{~W} 1 \mathrm{H}$ (What, When, Where, Who, Why, How) [3]. Với sự kết hợp này, Ogle đã bổ sung thêm cột $\mathrm{H}$ ở sau cùng - nội dung khuyến khích người học định hướng nghiên cứu. Sau khi người học đã hoàn tất nội dung ở cột $\mathrm{L}$, có thể các em muốn tìm hiểu thêm về một thông tin. Để thực hiện, các em sẽ nêu biện pháp để tìm thông tin mở rộng. Những biện pháp này sẽ được ghi nhận ở cột H (How I Learn/How can I Learn more) [4].

\section{Vận dụng kỹ thuật dạy - học KWLH}

\subsection{Vận dụng kỹ thuật dạy học KWLH trên lớp học}

Để thu hút sự tham gia của sinh viên một cách trực tiếp vào các hoạt động tư duy và giải quyết vấn đề theo tiêu chuẩn 8 của CDIO, chúng tôi đã sử dụng kỹ thuật KWLH. Việc vận dụng kỹ thuật dạy học này trên lớp học một cách hiệu quả nhất, giảng viên và sinh viên cần phải thực hiện các bước sau:

Bước 1: Chọn bài học/chủ đề. Giảng viên chọn chủ đề giảng dạy hoặc gợi ý cho sinh viên đọc hiểu hiệu quả.

Bước 2: Giảng viên vẽ sơ đồ KWLH lên bảng và sinh viên vẽ lên vở ghi chép của mình (cá nhân), vẽ lên giấy lớn (nhóm) như bảng 1 .

Bảng 1. So đồ kỹ thuật dạy - học KWLH

\begin{tabular}{|l|l|l|l|}
\hline $\begin{array}{l}\text { K - Cái đã biết } \\
\text { (What I Know) }\end{array}$ & $\begin{array}{l}\text { W - Cái muốn biết } \\
\text { (What I want to Know) }\end{array}$ & $\begin{array}{l}\text { L - Cái đã học được } \\
\text { (What I Learned) }\end{array}$ & $\begin{array}{l}\text { H - Cách tự học mở } \\
\text { rộng } \\
\text { (How can I Learn more) }\end{array}$ \\
\hline 1$).$ & $1)$. & $1)$. & $1)$. \\
$2)$. & 2 ). & 2 ). & 2 ). \\
$3)$. & $3)$. & $3)$. & $3)$. \\
---- & ----- & ---- & ---- \\
\hline
\end{tabular}

Nguồn: Hamdan, Mohammad Hussein (2014)

Bước 3: Giảng viên dẫn dắt vào bài học, gợi ý và đề nghị sinh viên công não nhanh tất cả những gì mà họ biết về chủ đề/bài học. Thông tin này được ghi vào cột $\mathrm{K}$ của sơ đồ bằng các từ, cụm từ. Để khuyến khích sinh viên chủ động tham gia vào quá trình học tập, 
giảng viên nên chuẩn bị những câu hỏi gợi ý để giúp sinh viên động não, bởi vì, có thể các em chưa kịp hồi tưởng những gì đã biết. Điều này cũng muốn nhắc nhở giảng viên đầu tư nhiều học cho bài giảng và không nên chỉ gợi ý bằng câu nói "Hãy nói ra nhũng điều mà các em biết về ...."

Bước 4: Giảng viên hỏi sinh viên xem các em muốn biết thêm điều gì về bài học/chủ đề. Nếu sinh viên đưa ra câu nói bình thường, giảng viên nên chuyển các câu đó thành câu hỏi và ghi nhận vào cột $\mathrm{W}$. Đây là bước quan trong giảng dạy, thể hiện sự tôn trọng ý tưởng của sinh viên cũng như hiểu được chuẩn đầu ra mà các em mong muốn. Vì vậy, để khuyến khích các em chủ động tìm hiểu và đi đúng trọng tâm bài học, người dạy nên gợi mở ý tưởng, hoặc chuẩn bị sẵn một số câu hỏi để bổ sung vào cột $\mathrm{W}$.

Bước 5: Giảng viên giảng bài, tổ chức các hoạt động khác cho sinh viên tham gia hoặc sinh tự đọc tài liệu theo hướng dẫn của giảng viên. Sau khi đã giảng xong bài học/chủ đề, nếu là bài đọc thì sau khi sinh viên đọc xong, giảng viên yêu cầu sinh viên tóm tắt những gì đã được học/đã đọc và tự điền câu trả lời cho câu hỏi ở cột $\mathrm{W}$ mà sinh viên tìm được (trong quá trình học tập/tự đọc). Thông tin từ các câu trả lời được ghi vào cột $\mathrm{L}$. Ngoài ra, chúng ta nên khuyến khích các em đánh dấu vào cột $L$ những điều các em cảm thấy tâm đắc nhất. Giảng viên tiếp tục điều phối cho người học thảo luận những thông tin ghi nhận ở cột $\mathrm{L}$, đây là cuộc thảo luận để sinh viên hiểu sâu bài học. Nhằm tăng cường tính hợp tác, làm việc nhóm tích cực, chủ động. Giảng viên có thể sử dụng các kỹ thuật dạy học khác để sinh viên thảo luận, củng cố nội dung chính của bài học/chủ đề.

Bước 6: Sau khi củng cố bài học, giảng viên khuyến khích sinh viên nghiên cứu thêm về những câu hỏi đã nêu ở cột $\mathrm{W}$ nhưng chưa tìm được câu trả lời từ bài học. Bước này, yêu cầu người học suy nghĩ cách giải quyết các câu hỏi, nêu biện pháp tìm kiếm nhằm mở rộng thông tin hay đưa ra định hướng tự học, tự nghiên cứu và vận dụng bài học vào thực tiễn. Những cách giải quyết này được ghi vào cột $\mathrm{H}$ của sơ đồ.

Quá trình vận dụng kỹ thuật KWLH trên lớp sẽ đánh giá trước được sự hiểu biết của sinh viên, tìm hiểu nhu cầu, sở thích của các em, cho các em được tham gia một cách tích cực, chủ động vào các hoạt động học tập trong quá trình triển khai giảng dạy, đánh giá sau quá trình tiếp thu bài học và cuối cùng để sinh viên chủ động tìm ra các hướng nghiên cứu, giải quyết vấn đề. Kỹ thuật này không chỉ giúp sinh viên đánh giá được kết quả học tập, thể hiện mong muốn trong tương lai của bản thân mà còn giúp giảng viên tự đánh giá phương pháp, năng lực giảng dạy của họ. Như vậy, triển khai kỹ thuật dạy - học này một cách hiệu quả sẽ giúp sinh viên đạt được Tiêu chuẩn 8 - học tập chủ động và có cơ sở để cải thiện, phát triển giảng viên theo Tiêu chuẩn 9 và 10 của đề xướng CDIO.

\subsection{Vận dụng kỹ thuật KWLH giúp sinh viên tụ học}

Kỹ thuật KWLH hoạt động như một chiến lược tự học/đọc hiểu hiệu quả. Chính vì vậy, việc hướng dẫn sinh viên sử dụng sơ đồ này vào quá trình tự tìm hiểu, tự học sẽ là công cụ thiết lập các mục tiêu/chuẩn đầu ra để đọc, tìm kiếm thông tin từ các nguồn tài liệu, sắp xếp thông tin đó thành các phác thảo đồ họa và viết tóm tắt dựa trên các phác đồ đó. Để sử dụng sơ đồ này, giảng viên và sinh viên cần thực hiện một số bước tương tự hình thức học tập ở trên lớp, một số bước thực hiện ở nhà. Các bước đó được thực hiện như sau:

Bước 1: Chọn chủ đề/ bài đọc/ chương và giao cho sinh viên. 


\section{Bước 2: Tạo bảng KWLH}

Bước 3: Khuyến khích sinh viên nêu ra những gì mà các em biết và ghi nhận và cột $K$.

Bước 4: Yêu cầu các em viết ra những điều mà các em muốn biết về chủ đề/ bài đọc. Thông tin này được ghi ở cột $\mathrm{W}$. Giảng viên cũng nên gợi ý một số vấn đề để các em tự đặt câu hỏi.

Bước 5: Giảng viên phát tài liệu hoặc hướng dẫn về nguồn tài liệu cần đọc để các em giải quyết các câu hỏi đã nêu ra ở cột $\mathrm{W}$. Sau đó giảng viên yêu sinh viên phải hoàn thành cột $L$, cột $H$ như một bài tập về nhà. Sinh viên tự học, tự đọc/tự tìm hiểu tài liệu để giải quyết vấn đề rồi hoàn thành cột $\mathrm{L}$ - những gì các em đã tìm kiếm được qua tài liệu, cột $\mathrm{H}$ định hướng tự nghiên cứu bài học này.

Bước 6: Sinh viên nộp sản phẩm. Sản phẩm này sẽ được giảng viên chấm điểm cá nhân. Sau đó, giảng viên hướng dẫn sinh viên thảo luận theo nhóm ngẫu nhiên do giảng viên sắp xếp để làm rõ, hiểu sâu hơn về bài học. Nội dung chính của bài học được nhóm tổng hợp trên sơ đồ tư duy, nhóm cử đại diện trình bày trước lớp.

Bước 7: Giảng viên giảng dạy, đúc kết lại nội dung văn bản và đánh giá quá trình tự học và làm việc nhóm của sinh viên.

Như vậy, dựa vào lý thuyết của kỹ thuật KWLH, mỗi giảng viên sẽ có một cách triển khai để nâng cao hiệu quả của việc dạy - học, tăng tính chủ động, tích cực học tập của sinh viên khi xây dựng chương trình đào tạo theo $\mathrm{CDIO}$. Với bản thân tác giả, sau nhiều lần áp dụng và thấy được hiệu quả mà kỹ thuật này mang lại, tác giả mạn phép khẳng định, sử dụng kỹ thuật dạy - học KWLH, giảng viên nhấn mạnh nhiều hơn vào việc tăng tính chủ động của sinh viên, thu hút sinh viên tham gia vào khám phá, ứng dụng, phân tích và đánh giá các ý tưởng của bài học để đạt được chuẩn đầu ra cao nhất.

\section{Kết luận}

Dạy - học tích cực, chủ động theo Tiêu chuẩn 8 của CDIO là yếu tố trọng tâm trong hệ thống giáo dục và KWLH là một một kỹ thuật giúp giảng viên phát huy được tính tích cực, chủ động của sinh viên. Kỹ thuật này đã thu hút sinh viên trong suốt quá trình dạy học, vì nó có dẫn dắt vào bài bằng chủ đề và tạo bảng, đánh giá trước ở cột $\mathrm{K}$, triển khai ở cột $\mathrm{W}$, đánh giá sau và tóm tắt ở cột $\mathrm{L}$ và gợi ý định hướng tự học, tự nghiên cứu cột $\mathrm{H}$. KWLH là kỹ thuật dạy - học được các nhà nghiên cứu đánh giá không chỉ có hiệu quả đối với việc giảng dạy trên lớp mà còn ứng dụng rất tốt đối với việc tự học, tự nghiên cứu của sinh viên. Chính vì vậy, kỹ thuật này cần được triển khai nhiều hơn, rộng hơn cho sinh viên để tăng tính chủ động học tập, nghiên cứu, có như thế mới đáp ứng được nhu cầu ngày càng cao của các nhà tuyển dụng. 


\section{Tài liệu tham khảo}

[1] http://www.cdio.org/implementing-cdio/standards/12-cdio-standards\#standard7, Truy cập ngày $24 / 12 / 2020$

[2] Sinambela, Erika, Sondang Manik, and Rotua Elfrida Pangaribuan (2015), "Improving students' reading comprehension achievement by using KWL strategy", English Linguistics Research 4.3, 2015, 13-29.

[3] Hamdan, Mohammad Hussein, "KWL-Plus Effectiveness on Improving Reading Comprehension of Tenth Graders of Jordanian Male Students", Theory \& Practice in Language Studies, 2014, 4.11.

[4] Zhang Fengjuan, "The Integration of the Know-Want-Learn (KWL) Strategy into English Language Teaching for Non-English Majors", Chinese Journal Of Applied Linguistics (Foreign Language Teaching \& Research Press), 2010, 33(4).

[5] Al-taie, S. H. K. (2010), "The Effect of Applying KWL Technique on Teaching ESP Students", Journal Of Educational and Psychological Researches, 7(27), 378-396.

[6] Andrew Marchand (2015), Instruction Skills Workshop, Tài liệu tập huấn các kỹ năng giảng dạy.

[7] Dương Minh Ngọc, Sử dụng kỹ thuật KWL trong dạy học bài nhị thức Niu-ton nhằm phát triển năng lực người học, Tạp chí thiết bị giáo dục, Số 115, 2015, 59-61 \& 62. 